\title{
Congenital tumors: imaging when life just begins
}

\author{
Leonor Alamo • Maja Beck-Popovic • \\ François Gudinchet • Reto Meuli
}

Received: 18 August 2010 /Revised: 17 November 2010 / Accepted: 27 January 2011 / Published online: 14 February 2011

(C) European Society of Radiology 2011

\begin{abstract}
Background The technical developments of imaging methods over the last 2 decades are changing our knowledge of perinatal oncology. Fetal ultrasound is usually the first imaging method used and thus constitutes the reference prenatal study, but MRI seems to be an excellent complementary method for evaluating the fetus. The widespread use of both techniques has increased the diagnosis rates of congenital tumors. During pregnancy and after birth, an accurate knowledge of the possibilities and limits of the different imaging techniques available would improve the information obtainable, thus helping the medical team to make the most appropriate decisions about therapy and to inform the family about the prognosis.

Conclusion In this review article, we describe the main congenital neoplasms, their prognosis and their imaging characteristics with the different pre- and postnatal imaging methods available.
\end{abstract}

Keywords Pediatric oncology Prenatal diagnosis .

Fetal US · Fetal MRI $\cdot$ Neonatal imaging

L. Alamo $(\bowtie) \cdot$ F. Gudinchet $\cdot$ R. Meuli

Department of Diagnostic Radiology, Unit of Pediatric Radiology, University Hospital of Lausanne (CHUV),

Rue du Bugnon, 46,

1011 Lausanne, Switzerland

e-mail: Leonor.alamo@chuv.ch

M. Beck-Popovic

Department of Pediatrics, Unit of Onco-hematology,

Centre Hospitalier Universitaire Vaudois (CHUV),

Rue du Bugnon, 46,

1011 Lausanne, Switzerland

\section{Introduction}

Tumors are considered congenital when detected during pregnancy or in the first 3 months of life $[1,2]$. Congenital tumors represent only $1.5-2 \%$ of all pediatric tumors, with a prevalence of $1: 12,500$ to $1: 27,500$ live births [3-5]. Recent studies demonstrate an increase in the incidence of congenital tumors [6], probably as a consequence of both the generalization of routine prenatal and neonatal medical controls and of the improvement of prenatal imaging techniques.

Congenital tumors differ in many aspects from tumors presenting in older children, with respect to histology, behavior and prognosis. The most frequent congenital tumor types are shown in Table 1 [2, 3, 5-8]. The diagnosis of a congenital tumor involves a tremendous emotional impact for a family and implies ethical questions about prognosis, therapy possibilities and long-term consequences of the treatment. Prognosis is related not only to the histological type, but also to the size and location of the tumor and the presence or not of associated congenital anomalies, which are found in $15-20.8 \%$ of cases $[5,9]$. Another prognostic factor is the age at which the lesion is detected, with most histological tumor types showing a worse prognosis for cases detected during pregnancy [10-14].

Over the last few years, we have witnessed significant technical improvements in prenatal imaging diagnostic tools. Ultrasound is usually the first imaging method used, but antenatal magnetic resonance imaging (MRI) is increasingly being used as a complementary study. Standard postnatal imaging methods include conventional X-ray, ultrasound, CT and MRI.

The purposes of this review are to describe the most frequent types of congenital tumors and their prognosis, to review the main imaging characteristics of this heterogeneous 
Table 1 Distribution of main congenital tumors [2, 3, 5-8]

\begin{tabular}{ll}
\hline Type of tumor & $\%$ \\
\hline Extracranial teratomas & $23.5-29 \%$ \\
Neuroblastomas & $22.5-30 \%$ \\
Soft tissue tumors & $8.1-12 \%$ \\
CNS tumors & $5.9-10 \%$ \\
Leukemia & $5.9 \%-12.35 \%$ \\
Renal tumors & $5-7.1 \%$ \\
Hepatic tumors & $5 \%$ \\
Cardiopulmonary tumors & $<3 \%$ \\
\hline
\end{tabular}

group of neoplasms, and to define the role of the different preand postnatal imaging methods in the characterisation of congenital tumors, with special emphasis on prenatal MRI.

\section{Extracranial teratomas}

Extracranial teratomas are neoplasms derived from the primordial germ cells. They may contain components arising from all three germ layers and therefore may present different tissue types. Most congenital teratomas are histologically benign and typically located mid-line. The main location of these tumors is the sacrococcygeal region ( $45 \%$ of cases), followed by the cervicofacial area $(28 \%)$ and the thorax (11\%) [11].

Sacrococcygeal teratomas (SCT) are one of the most common overall congenital neoplasms, with an incidence of 1:40,000 infants and a female predominance of $4: 1$ [15, 16]. They arise from rests of pluripotent cells at the caudal end of the notochord, originating from the coccyx. Of all teratomas, these tumors are associated with the highest incidence of malignancy (10\%) and of associated anomalies (10-15\%). According to the tumor extension, SCTs are classified into four categories by the American Pediatric Surgery Association: type I, lesions almost completely external; type II, predominantly external masses with pelvic extension; type III, external tumors with intra-abdominal extension; type IV, exclusively intra-abdominal lesions [17]. Fetal tumors are usually large exophytic masses (Fig. 1a, b) with a high incidence of complications, such as polyhydramnios, fetal cardiac failure, fetal hydrops, tumoral hemorrhage and prematurity [11], which are related to the tumor size and to the effect of the intrapelvic and intra-abdominal tumor components over the developing organs [18]. The differential diagnosis includes lipoma, dermoid and lumbosacral myelomeningocele [19].

Cervicofacial teratomas (Fig. 1c, d) have an incidence of $1: 40,000$ to $1: 80,000$ live births. They may originate in different anatomical structures, including the orbit, the naso- and oropharynx, the tongue, the palate and the anterior neck [19]. Cervical teratomas in utero may not only cause hydrops secondary to fetal swallowing disturbances, but also fetal pulmonary hypoplasia. Large tumors may cause hyperextension of the fetal neck with resulting dystocia [20]. Ex utero intrapartum treatment, or EXIT, has been performed in recent years in cases of giant neck masses in highly specialized neonatal medical centers, improving the initial fetal survival [21, 22].

Thoracic teratomas originate mainly in the pericardium [11]. Pericardial effusion is typically present and may lead to life-threatening tamponade. Indeed, in cases of fetal pericardial effusion, teratoma should be seriously considered in the differential diagnosis. Pericardiocentesis can help to prevent fetal death. Mediastinal teratomas are rare. They should be removed after birth to prevent malignant degeneration [23].

Congenital sacrococcygeal and cervicofacial teratomas present an overall mortality of $30-33.5 \%$, mainly related to large tumors with extensive local infiltration [11]. The main role of pre- and postnatal imaging for teratomas is to recognize any associated anomalies and to determine the full extent of the mass for prognosis and evaluation of surgical planning. On fetal ultrasound, teratomas are seen as large, heterogeneous masses due to their different tissue components. In some cases of SCT, an accurate ultrasound evaluation may be insufficient because of shadowing by the fetal pelvic bones, which may cause an underestimation of the tumor [18]. Fetal MRI is therefore considered the method of choice for evaluating the extent of the internal mass (Fig. 1a). Indeed, the method can serve as a roadmap before surgery, as ultrasound images are sometimes difficult to interpret for clinicians and may fail to provide a good overview of the limits of the relation between the tumor and the surrounding anatomy. Post-natal CT and MRI studies show a heterogeneous mass with solid, liquid and fat components (Fig. 1d), and variable contrast enhancement. Calcifications and bone tissue are often found and are better recognized on CT (Figs. 1, 2b).

\section{Neuroblastoma}

The neuroblastoma is the second most common pediatric malignancy overall, after teratoma, in most published series $[3,5]$ and the most common malignancy of the first month of life (30-50\%] [8]. The tumor arises from primordial neural crest cells, anywhere along the sympathetic chains, at the adrenal medulla (Fig. 2b), the extra-adrenal retroperitoneum (Fig. 2c, d) or the posterior mediastinum.

Fetal neuroblastoma is usually detected during the third trimester of pregnancy. It is mostly of adrenal origin $(90 \%$ of cases). The prognosis is excellent, with a survival rate of 
Fig. 1 Extracranial teratomas. Case 1. SCT. Coronal (a) and sagittal T2-TRUFI (b) fetal MRIs (29th week) show the large, complex SCT type II with both cystic (arrow) and solid components. The images clearly illustrate the extension of the tumor into the pelvis, the abdomen and the spinal canal (block arrow). Case 2. Cervical teratoma (3 months old). The CT topogram (c) and the axial NECT image (d) demonstrate the heterogenicity of the tumor, with calcifications (white arrows), fat, solid and liquid (star) components
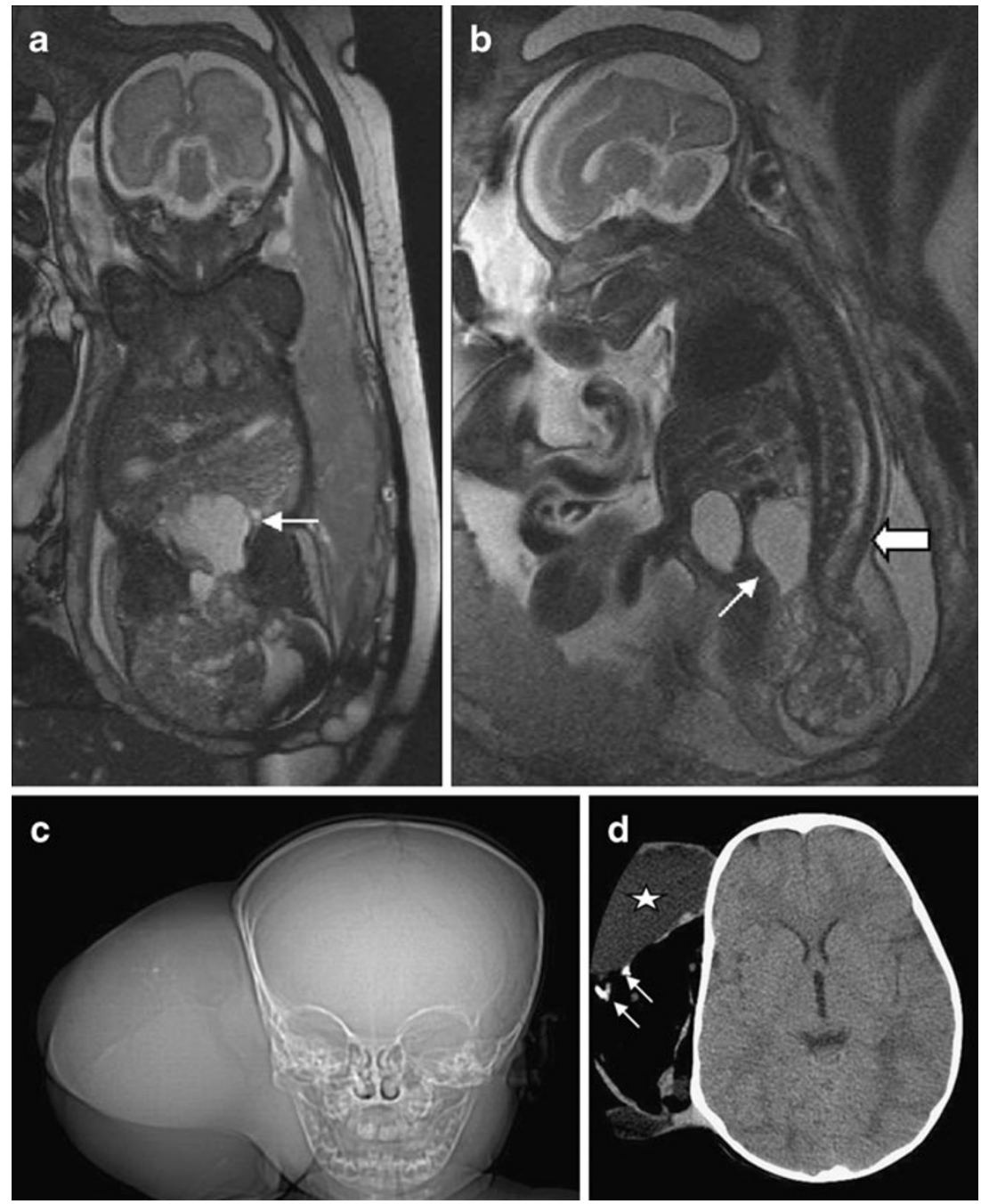

88-90\% [1, 24], metastases are rare and spontaneous involution in utero has been described. On ultrasound, the tumor is mostly seen as a solid, heterogeneous echogenic mass that displaces the adjacent kidney inferiorly and laterally. Cystic tumors are often seen and have an excellent prognosis [24]. Calcifications are present in 30\% of all cases. On MRI, neuroblastomas are typically heterogeneous, with relatively low signal intensity on T1- and high signal intensity on T2-weighted images. Cystic changes appear as bright areas on T2-weighted images [8].

Neonatal neuroblastomas are of adrenal origin in $45 \%$ (Fig. 2b) and of extra-adrenal origin in 55\% of all cases (Fig. 2c, d]. Although often metastatic [60\% of cases, principally liver (Fig. 2c), bone and skin], it has an overall survival rate of $64 \%[1,24]$. On CT, the tumor is heterogeneous, with calcifications, necrosis and areas of hemorrhage. Extension into the spinal canal is seen in $10 \%$ of abdominal (Fig. 2d) and in $28 \%$ of thoracic masses, and is clearly visualized on MRI studies [8].
Metaiodobenzylguanidine (MIBG- $\mathrm{I}^{123}$ ) scintigraphy is an excellent method for determining the presence of metastases or tumor recidivation, but $30 \%$ of neonatal tumors are MIBG-negative [25].

The International Neuroblastoma Staging System [26] is mainly based on the degree of surgical resection and shows some difficulties for application in congenital cases. Recently, a new International Neuroblastoma Risk Group Staging System (INRGSS), based on imaging findings, was designed to stage the patients at the time of diagnosis. In this classification, the extent of locoregional disease is determined by the absence (L1) or presence (L2) of imaging-defined risk factors [27], with stage M used for widely disseminated disease and stage MS for metastatic disease limited to liver, skin and bone narrow in children younger than 18 months [28]. The main role of imaging methods for both fetal and neonatal neuroblastoma is then to determine the origin of the mass, its locoregional extent and the presence or not of metastases for staging before therapy. 
Fig. 2 Neuroblastoma. Case 1 . Typical neuroblastoma. The abdominal ultrasound (a) and the axial fat-saturated T2-weighted images (b) show the welldefined, homogeneous mass arising from the right adrenal gland (white arrow) and displacing the kidney in this 2-day-old baby. Case 2. Axial contrast-enhanced CT (CECT) (c) shows the enhancing retroperitoneal tumor (white arrow) originating from the organ of Zückerkandl in this 10-day-old girl. Note the hepatic infiltration (arrow). Case 3. Axial CECT (d) shows the mass arising from the right paraspinal sympathetic chain and extending into the spinal canal in this 3-day-old baby boy. Note the enlargement of the spinal canal (white block arrow). Case 4. Atypical neuroblastoma. Axial ultrasound (e) and T1-weighted images after i.v. contrast medium administration (f) show the retroperitoneal, lobulated mass infiltrating the right kidney and causing hydronephrosis (arrow) in this 5-week-old baby. The lesion was initially considered to be a renal tumor, but histology revealed neuroblastoma
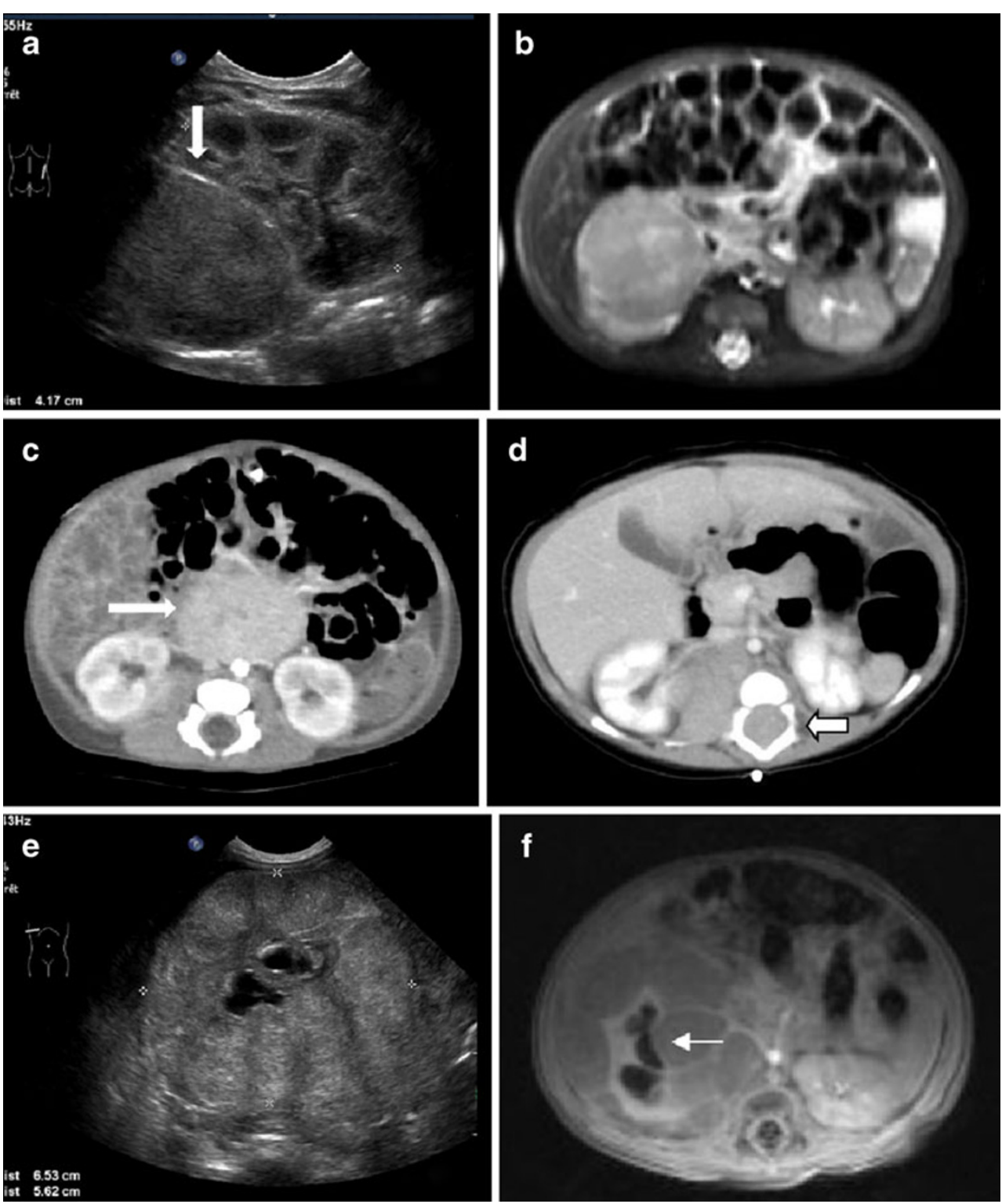

\section{Soft-tissue tumors}

Congenital soft-tissue tumors constitute a heterogeneous group of lesions, including mainly vascular and muscular tumors. The International Society for the Study of Vascular Anomalies (ISSVA) differentiates between vascular tumors and vascular malformations (capillary, venous, lymphatic or mixed) [29], which are not discussed in this review.

Infantile hemangiomas $(\mathrm{IH})$ are true neoplasms that originate from the cellular proliferation of vascular endothelial cells. They account for a significant number of prenatally diagnosed neoplasms [30], but because most of them are small or absent at birth, they are often not included in the register of congenital tumors [1]. The most frequent location is the head and neck (60\%) (Fig. 3b). IH usually present a biphasic cycle, with initial rapid growth followed by an involution over months or years. Most $\mathrm{IH}$ require neither therapy nor imaging studies. On ultrasound, they appear as lobulated, hypo- or hyperechoic, welldefined masses with increased Doppler flow.

The notion of congenital hemangiomas has been introduced recently, differentiating between non-involuting congenital hemangiomas, or NICH, and rapidly involuting congenital hemangiomas, or RICH [31]. Although both RICH and NICH groups show similar imaging findings to $\mathrm{IH}$ on ultrasound, they may also present vascular aneurysms, intravascular thrombi and arteriovenous shunting, features not usually observed in IH [32]. Large congenital tumors may cause compression of vital structures or cardiovascular complications, and in these cases, MRI is the method of choice to determine the mass extension and its relationship to the adjacent anatomical structures 

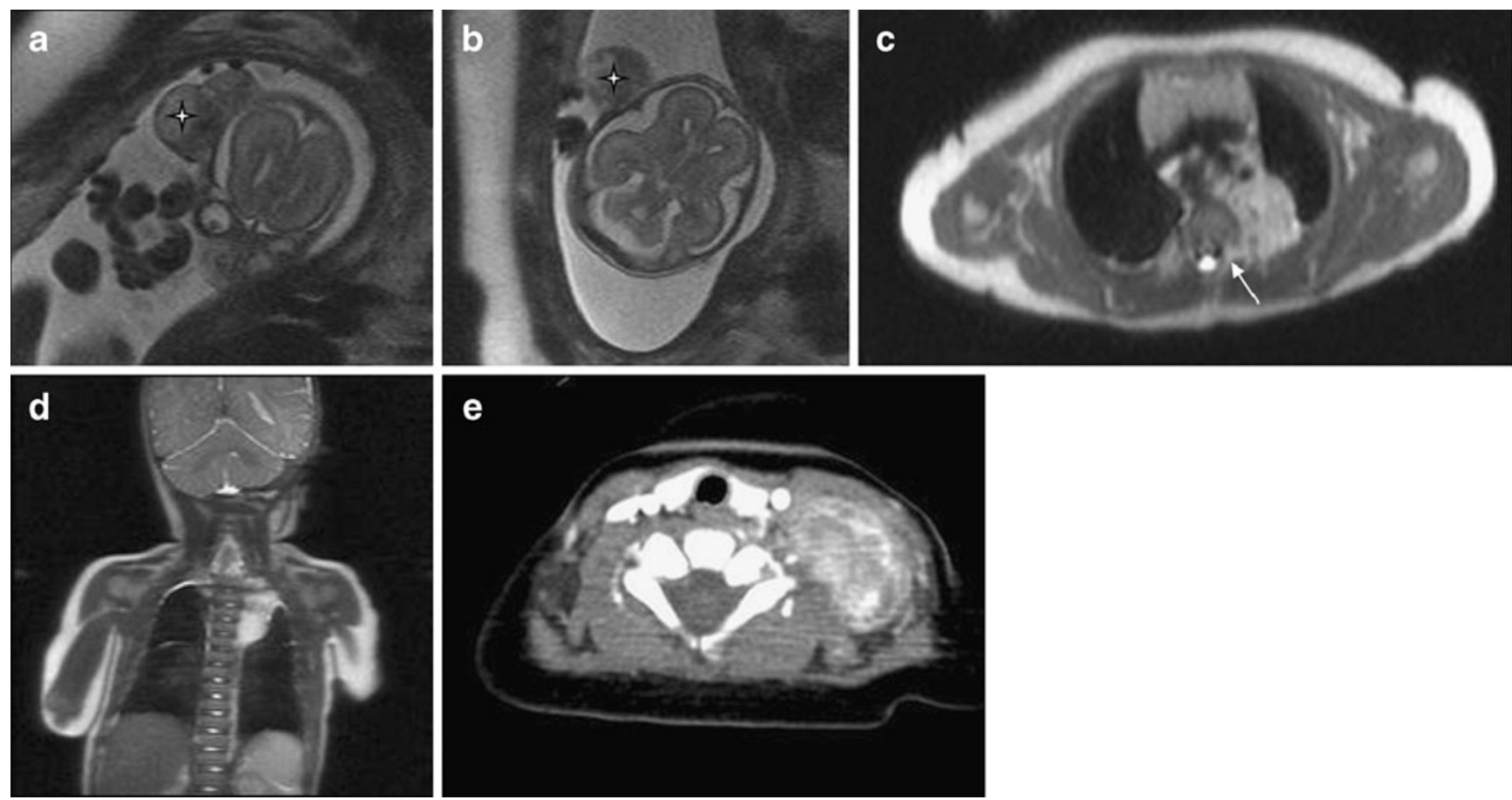

Fig. 3 Soft-tissue tumors. Cases 1 and 2. Congenital hemangiomas. Coronal (a) and axial (b) fetal T2-HASTE MRI (21st week) demonstrate the cephalic, but completely extracranial heterogeneous mass (star). The axial (c) and coronal (d) T2-weighted images show the homogeneous hyperintense mass in the left posterior mediastinum, extending into the spinal canal (arrow) in this newborn. The mass was biopsied to exclude a neuroblastoma. Case 3. Cervical myofibromatosis in a 2-month-old boy. Axial CECT image (e) shows the large, infiltrating left cervical muscular mass, with intense enhancement after contrast medium administration
(Fig. 3c, d). Hemangiomas are usually hyperintense on T2-weighted and isointense on T1-weighted images related to muscle, with prominent draining veins [5] and intense enhancement after contrast medium administration [33], but no perilesional edema.

Fibrous connective tissue tumors are the largest group of congenital soft-tissue tumors. Fibromatosis and myofibromatosis are rare benign disorders with a tendency to infiltrate adjacent tissues but no metastases. They present an increment of the size and the number of lesions during the first year of life with later regression. The ultrasound shows a soft-tissue mass with variable echogenicity. They are muscle isodense and poorly defined on CT (Fig. 3d), with low signal intensity on both T1-weighted and T2-weighted images [34], infiltration of the fat and muscle, and intense enhancement after i.v. contrast medium injection on MRI. The malignant variant is the congenital infantile fibrosarcoma [1].

Rhabdomyosarcoma arises from the embryonic mesenchyme with the potential to differentiate into skeletal muscle. The tumor represents $>50 \%$ of all soft tissue sarcomas in children, but its congenital presentation is extremely rare [35]. It can arise in any anatomical region of the body (except bone) whether there is skeletal muscle or not, but most often it presents in the head and neck $(28-40 \%)$ and in the genitourinary region $(20 \%)$. The ultrasound shows a softtissue mass, similar to muscle, whereas MRI reveals a mass isointense to muscle on T1-weighted and hyperintense on T2-weighted images, with heterogeneous enhancement after gadolinium administration. Some previously classified congenital rhabdomyosarcomas with a bad outcome were probably unrecognized rhabdoid tumors. Immunochemistry tests show that rhabdoid tumors typically lack staining for BAF47 in tumor cells because of a clonal mutation in the INI1 gene [36]. This anomaly now allows correct differentiation between rhabdoid tumors - usually associated with a bad prognosis - and rhabdomyosarcomas [37].

The main role of imaging in the case of congenital soft tissue tumors is to determine the extent of the mass and to identify the invasion of adjacent structures in order to optimize the surgical approach.

\section{Central nervous system tumors}

Congenital tumors of the central nervous system (CNS) are rare. They have a poor prognosis with an overall mortality of $72 \%$ [37-41]. They represent only $0.5-1.9 \%$ of all pediatric brain tumors, but are responsible for $5-20 \%$ of deaths secondary to neoplasms in this age group [6,38].

Congenital CNS tumors differ in histology, location, biological behavior and response to therapy compared with CNS tumors in older children. The most frequent tumor 
types and their mortality rates appear in Table 2. Most congenital tumors (about 60\%) are supratentorial, arising from the pineal gland, the suprasellar area or the cerebral hemispheres. Macrocephaly is usually the first clinical finding. Increased intracranial pressure signs are not often observed because of the great capacity for accommodation to the increased volume in the fetus and in neonates with opened sutures [35]. Intratumoral hemorrhage is relatively common, varying between 3 and $18 \%$ [38-40].

Teratoma is the most common congenital brain tumor and represents 26.6 to $48 \%$ [38-41] (Table 2). Congenital teratomas are predominantly supratentorial, with the cerebral hemispheres being the main primary site, followed by the third ventricle and the pineal region [42]. On ultrasound, they are seen as typically large, mid-line heterogeneous tumors with solid areas replacing much of the brain. Cystic components are often found [41] and probably represent necrotic areas in tumors with a rapid growth rate. Calcifications are possible, but unusual.

The astrocytoma is the most common neuroglial tumor. The cerebral hemispheres are the main primary site, followed by the optic nerve, the thalamus and the mesencephalus. They typically present with macrocephaly. Fetal ultrasound and MRI show a solid tumor replacing the normal brain parenchyma. Hemorrhage is common.

The choroid plexus papilloma is composed of mature epithelial cells derived from choroid plexus epithelium. It presents clinically as macrocephaly, with rapid onset of hydrocephalus, secondary to the overproduction and/or mechanical obstruction to cerebrospinal fluid (CSF) circulation [1]. These are well defined, intraventricular masses, hyperechoic on ultrasound and mostly hyperattenuating on CT (Fig. 4a, b), with intense contrast enhancement. On MRI, they present as homogeneous masses on T1-weighted images, with the central tumor areas hypointense compared with grey matter on T2-weighted images [43]. Complete surgical resection is curative, but intraoperative hemorrhage is common and may have serious consequences. Choroid plexus carcinomas are very rare in neonates. They are often associated with marked vasogenic edema and may be hyperdense on CT because of the high cellularity.

Table 2 CNS tumors. Histological type and mortality rate of main CNS tumors [37-41]

\begin{tabular}{lll}
\hline CNS tumors & $\%$ & Mortality rate \\
\hline Intracranial teratomas & $26.6-48 \%$ & $88 \%$ \\
Astrocytomas & $7.4-28.8 \%$ & $64-68 \%$ \\
Choroid plexus papilloma & $3.7-13.2 \%$ & $27 \%$ \\
PNET & $3-13 \%$ & $88 \%$ \\
Craniopharyngioma & $5.6-6.8 \%$ & $76.5 \%$ \\
Ependymoma & $4.4 \%$ & $91 \%$ \\
\hline
\end{tabular}

Primitive neuroectodermal tumors (PNET) (Fig. 4c, d) are small-cell malignant tumors arising from the neural crest [40]. The most common locations are the cerebellum and the cerebral hemispheres. Congenital tumors have a poor prognosis as a consequence of the rapid tumor growth, with early extension throughout the CSF pathways.

The diagnosis of fetal and neonatal brain tumors is associated with high mortality rates (Table 2). Recent advances in MRI technology include the development of magnetic resonance diffusion sequences (DWI), which may identify areas of restricted water movement and of magnetic resonance spectroscopy (MRS), which recognizes changes in metabolite concentration [44]. These technical improvements have opened up exciting perspectives, suggesting the possibility of differentiating between histological tumor types [20,45], but preliminary reports have not yet been confirmed for congenital brain tumors. The main role of imaging studies is still to determine the extent of the tumor in order to evaluate therapy challenges and to identify potentially curable tumors, such as plexus papillomas, differentiating them from rapidly fatal ones.

\section{Renal tumors}

Renal tumors represent $5-7.1 \%$ of all congenital tumors (Table 1). They are mostly benign, with the most frequent histological types being mesoblastic nephroma (MSN), nephroblastomatosis and the multilocular cystic nephroma [14]. Wilms' tumor and renal rhabdoid tumors are rare in neonates $[1,14,46]$.

The MSN represents $66 \%$ of all congenital renal tumors [14]. It is a benign hamartomatous tumor, arising from proliferating nephrogenic mesenchyme. Fetal tumors have a worse prognosis than neonatal ones [14]. They present in utero as large, solid, infiltrative, unilateral renal masses [47], sometimes associated with polyhydramnios. Hypercalcemia has been described in neonatal cases $[1,3]$. The tumor has variable echogenicity, with homogeneous solid tissue on ultrasound. CT and MRI show a solid, relatively homogeneous renal tumor, typically involving the renal sinus and with variable contrast enhancement (Fig. 5a, b). The mass may present cystic and hemorrhagic areas [46].

Nephroblastomatosis consists of diffuse, multifocal nephrogenic rests in the kidneys. It is found incidentally in $1 \%$ of normal children at post-mortem studies and may cause a Wilms' tumor in $30-40 \%$ of the cases (Fig. 5c). There is a well-known association with Beckwith-Wiedemann syndrome, trisomy 18 and sporadic aniridia [48]. Typical ultrasound images show multifocal, subcapsular renal nodules, hypo- or isoechoic, related to renal parenchyma. The nodules present low attenuation on $\mathrm{CT}$ and low signal intensity on both T1- and T2-weighted images on MRI 
Fig. 4 Intracranial tumors. Case 1. Choroid plexus papilloma. Axial T2-weighted fetal MRI (34th week) (a) shows an intraventricular, solid mass in the posterior horn of the right lateral ventricle (white arrow). Postnatal axial T2-weighted image (b) 3 days after birth reveals an increase in the tumor size. Note the extensive paraventricular edema. Case 2. PNET. Sagittal T1-weighted image after i.v contrast medium administration (c) and coronal T2-weighted image (d) in this 6-week-old boy presenting with macrocephaly show the heterogeneous tumor in the posterior fossa (arrow), with both solid and liquid (block arrow) components. The tumor causes a secondary hydrocephalus
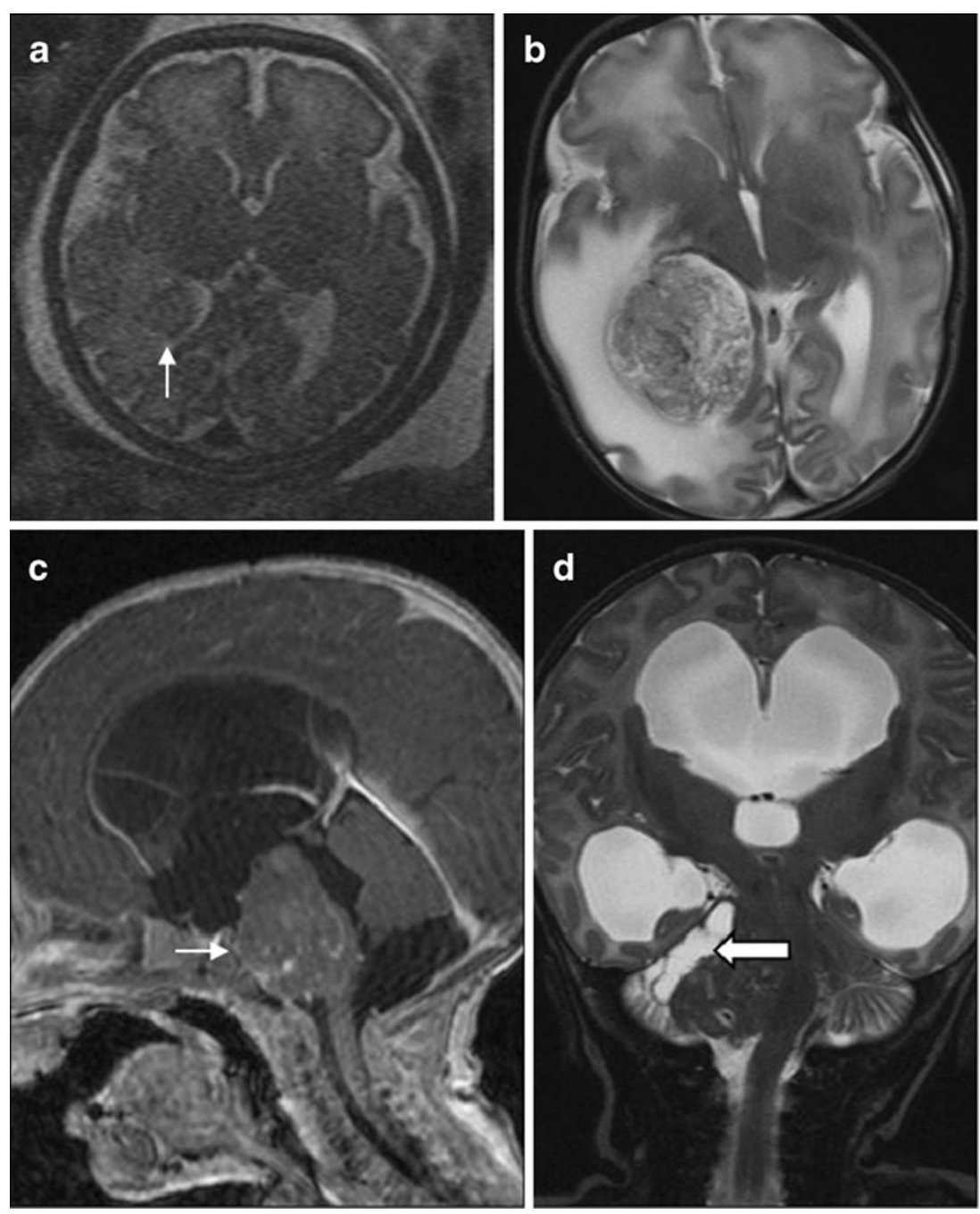

relative to normal parenchyma, with reduced contrast enhancement.

Multilocular cystic nephroma (MCN) is a benign cystic renal neoplasm, arising from metanephric blastema. Imaging shows a large multilocular cystic renal mass, with septa as the only solid component (Fig. 5d). Septa may enhance after contrast medium administration. Metastatic disease has not been reported.

The role of imaging studies for congenital renal tumors is to determine the extent of the mass and its relationship to the renal vascular structures before surgical therapy.

\section{Hepatic tumors}

Hepatic tumors comprise 5\% of all congenital neoplasms (Table 1). Excluding metastases, principally from leukemia and neuroblastoma [1], most primary hepatic tumors are hemangiomas $(60.3 \%)$, followed by mesenchymal hamartoma $(23.2 \%)$ and hepatoblastoma (16.5\%) [13].
The infantile hepatic hemangioma, frequently referred to as hepatic hemangioendothelioma, is the most common congenital vascular hepatic tumor $[13,49]$. It is a benign lesion composed of large endothelial-lined vascular channels. The clinical and biological behavior show initial proliferation and subsequent involution [50]. Hepatic hemangiomas may be focal (solitary) or multifocal, confluent lesions (Fig. 6a). Although spontaneous involution is possible, rapid growth may instigate fetal anasarca (Fig. 6b), thrombocytopenia resulting from consumptive coagulopathies (KasabachMerritt syndrome) or postnatal death due to cardiac failure [23]. On ultrasound, focal hemangioma is classically a well-defined, hypo-, iso- or hyperechogenic lesion with a prominent high flow on Doppler ultrasound [47]. Calcifications are common. Large tumors present as welldefined, solid masses, often with central necrosis. Most lesions are hypodense compared with the rest of the liver on non-enhanced CT [49]. After contrast medium administration, they show a centripetal enhancement that acquires uniformity on delayed CT. On MRI, the lesions 
Fig. 5 Renal tumors. Case 1 . Mesoblastic nephroma. Axial CECT (a) and fat-saturated T1-weighted image after i.v. contrast medium administration (b) show a solid, focal renal mass replacing the normal right renal parenchyma (block arrows) in this newborn boy. Case 2. Nephroblastomatosis with bilateral Wilms' tumors. Axial CECT (c) shows the welldefined bilateral renal masses (stars) in this 3-month-old baby, corresponding to a histologically proven bilateral Wilms' tumor in a child with areas of nephroblastomatosis. The masses remain hypodense compared with normal renal parenchyma. Wilms' tumors are very rare in fetuses and neonates. Case 3. MCN. T2-HASTE MR image (d) shows a multilocular cystic mass in the right kidney with tumor septae. Observe a similar but smaller left renal lesion (white arrow) in this 10-week-old boy with multiple congenital tumors (same patient as in Fig. 6c)
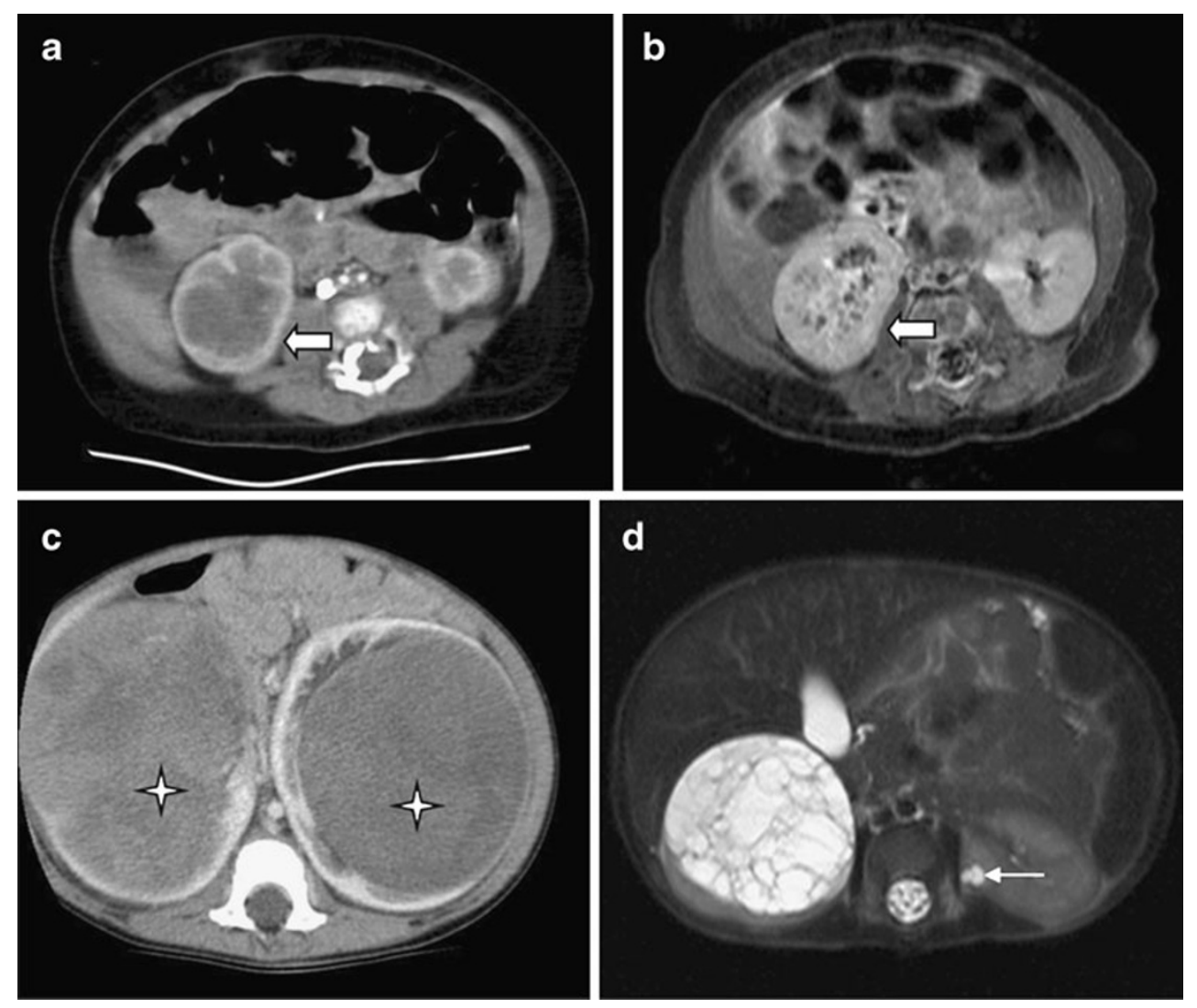

usually show low $\mathrm{T} 1$ and high $\mathrm{T} 2$ signal intensities, but may be heterogeneous because of hemorrhage and calcification [49], with variable enhancement patterns. Necrotic or thrombotic regions show a heterogeneous signal both before and after gadolinium administration.

The mesenchymal hamartoma is a developmental cystic tumor, resulting from the benign overgrowth of mature hepatic tissue, with variable loose mesenchyme. Association with Beckwith-Wiedemann syndrome has been reported. Ultrasound shows a tumor composed of variably sized anechoic cysts with internal septations. Solid tumors have been described, but they are atypical $[47,51]$. On MRI the cysts are fluid isointense lesions with septa and stromal components (Fig. 6c) [52].

Congenital hepatoblastoma is a malignant embryonic tumor composed of only epithelial cells or a mixture of epithelial and mesenchymal cells. They present as lobulated, sometimes multifocal large solid masses, most often in the right hepatic lobe $(60 \%)$. An association with hemihypertrophy (2\%), Beckwith-Wiedemann and intestinal polyposis is well established. The $\alpha$-fetoprotein may be negative in a considerable percentage of fetal and neonatal tumors [13]. Ultrasound shows a well-defined, solid mass (Fig. 6d), often with a spoked-wheel appearance. The lesion is heterogeneous, with calcifications $(50 \%)$, cystic and necrotic areas [47]. CT and MRI show a heterogeneous solid hepatic lesion with non-uniform enhancement.
The differential diagnosis of congenital hepatoblastoma is the malignant hepatic rhabdoid tumor. The most extensive published series about congenital hepatic tumors describes a poor prognosis for hepatoblastomas, with common early metastases and high mortality rates [13], but this series probably includes some cases of congenital rhabdoid tumors, an aggressive lesion previously classified as hepatoblastoma with negative $\alpha$-fetoprotein. As mentioned elsewhere in this article, immunochemistry tests for INI1/BAF47 protein, which is abnormally low in all rhabdoid tumors, have recently allowed a correct differentiation between the entities [36, 37, 53, 54], which present similar findings on imaging studies.

The main role of imaging methods for congenital hepatic tumors is to evaluate the anatomical extent of the tumor and to clarify the relationship with hepatic lobar anatomy before surgical planning.

\section{Cardiopulmonary tumors}

The heart is one of the most common organs of origin of congenital tumors, with a reported incidence of 0.14 to $0.2 \%$ [55]. The incidence has been increasing in recent years because of the technical advances of imaging studies, especially ultrasound cardiography and cardiac MRI. Cardiac tumors may be related to genetic disorders such 
Fig. 6 Hepatic tumors. Case 1 . Multiple hepatic hemangiomas. Coronal T2-HASTE MRI (a) shows the multiple hyperintense hepatic lesions (white arrows). Observe the right hemihypertrophy in this newborn with Beckwith-Wiedemann syndrome (vertical arrows). Case 2.

Hemangioendothelioma. Coronal (b) T2-HASTE MRI (27th week) shows a large and infiltrating mass arising from the left hepatic lobe with predominantly high signal intensity. Observe the signs of fetal congestive heart failure with marked hydrops fetalis (white block arrows). Case 3. Mesenchymal hamartoma. Axial T2-weighted image (c) shows a centrally located, hepatic hyperintense cystic mass with internal septations in this 10-week-old boy (same patient as in Fig. 5d, e). Case 4. Hepatoblastoma.

Abdominal ultrasound (d) shows the large, heterogeneous mass in the right hepatic lobe of this 2-day-old girl
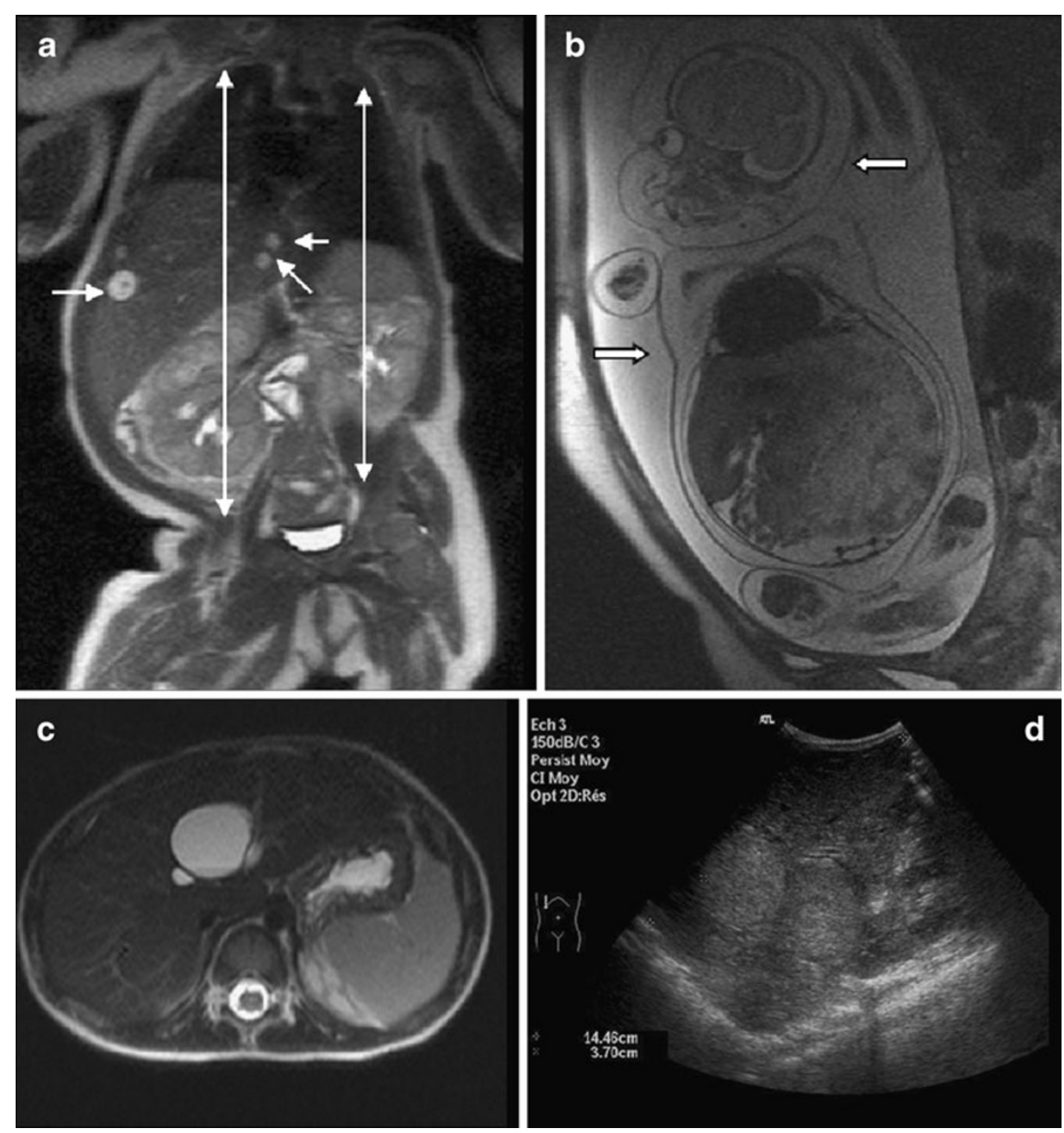

as neurofibromatosis, Beckwith-Wiedemann syndrome, familial myxoma syndrome and tuberous sclerosis. Most fetal and newborn cardiac tumors are benign. The most frequent cardiac tumor is the myocardial rhabdomyoma (78\%), followed by the pericardial teratoma (18\%) and the cardiac fibroma (12\%) [12]. Pericardial teratoma has already been described in this article.

The rhabdomyoma is a hamartoma, and as such it is not considered a true neoplasm. Its incidence oscillates between $0.12 \%$ in prenatal fetal studies and $0.02-0.08 \%$ in live-born infants $[55,56]$. The tumor is multiple in $>90 \%$ of cases, not invasive and not metastasizing [12]. Rhabdomyoma shows a high association with tuberous sclerosis $(86 \%)$ and may manifest as the earliest clinical sign of this pathological condition in utero. Indeed, the detection of multiple cardiac rhabdomyomas on prenatal ultrasound is considered sufficient to establish the diagnosis of tuberous sclerosis [57] and makes MRI evaluation of the fetal CNS mandatory [58]. Clinical findings oscillate from asymptomatic to congestive heart failure, arrhythmias, valvular dysfunction and sudden death, which cause a mortality of $40 \%$. Surgical intervention is only recommended in cases of persistent arrhythmias or severe hemodynamic compromise because rhabdomyomas usually increase in size until late pregnancy and then tend to regress progressively during the first year of life. Rhabdomyomas have been detected as early as the 19th week of pregnancy. They appear as rounded and homogeneous hyperechogenic areas (Fig. 7a), originating from the ventricular septum or the ventricular wall. Cardioultrasound is the imaging method of choice and provides a dynamic evaluation of the myocardial wall motion and the valvular functionality.

Pleuropulmonary congenital neoplasias are extremely rare. The pleuropulmonary blastoma is a primary mesenchymal neoplasm with a very poor prognosis. On prenatal ultrasound and MRI, it presents as a solid heterogeneous mass originating from the pleura or the pulmonary parenchyma (Fig. 7b), often with additional pleural effusion (Fig. 7c). After birth, the tumor may manifest as a benignappearing, air-filled cystic lesion or as large heterogeneous 

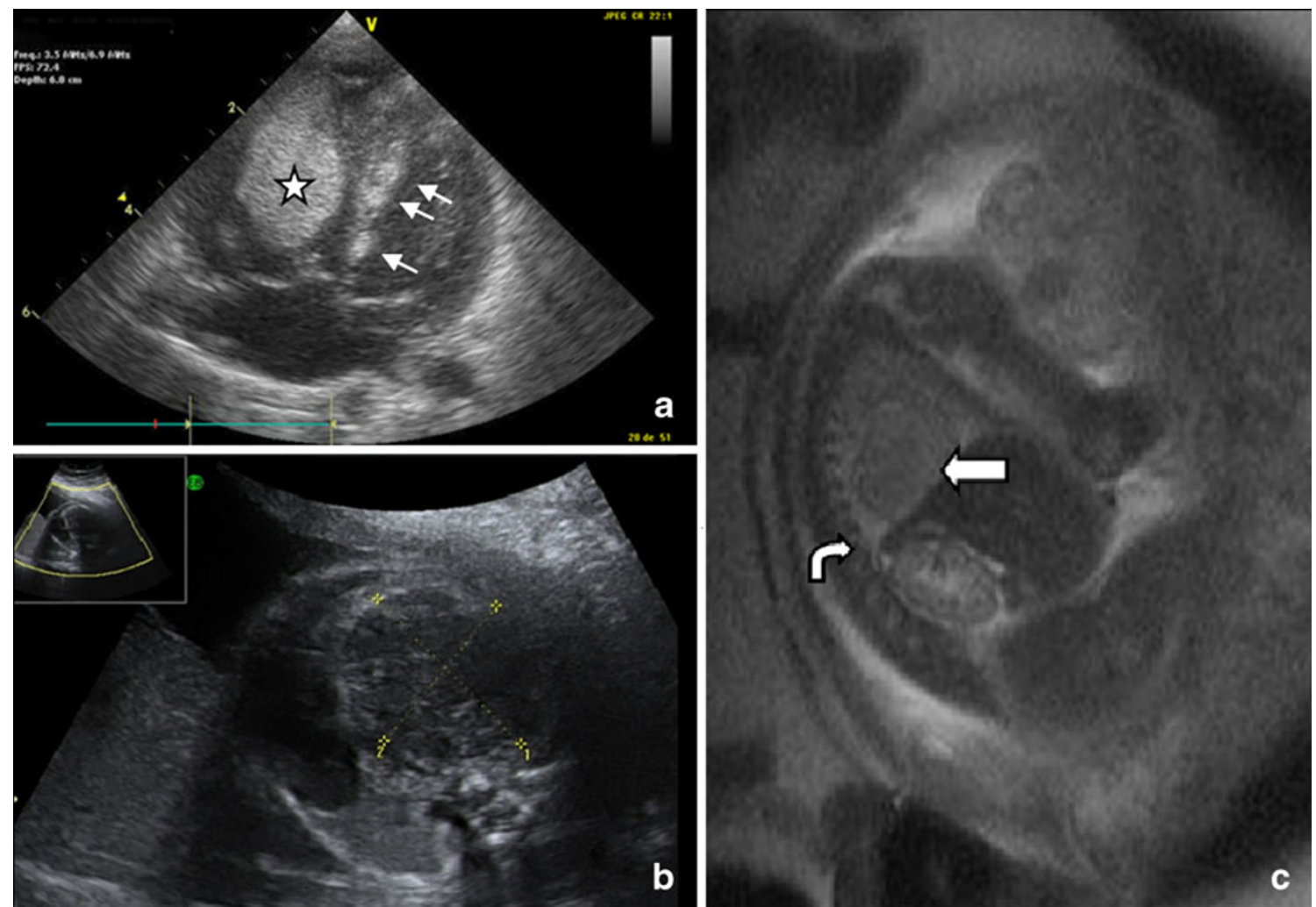

Fig. 7 Cardiopulmonary tumors. Case 1. Rhabdomyoma. The cardioultrasound image (a) shows the multiple hyperechoic tumors originating from the ventricular septum (arrows) and the ventricular walls. Observe the large mass in the right ventricle (star) of this asymptomatic 3-day-old newborn boy with tuberous sclerosis. Case 2.

solid masses. The main differential diagnoses include the rhabdomyosarcoma and the undifferentiated sarcoma.

\section{Conclusion}

Congenital tumors are a unique, heterogeneous group of neoplasms that differ in many aspects from tumors presenting later in life. Congenital fetal and neonatal neuroblastomas are associated with a better prognosis than tumors detected later in life, but they remain an exception. Although mostly benign, the therapeutic possibilities of most congenital tumors may be limited because of the tumor size, its location or the stage of the pregnancy at the time of diagnosis. Moreover, the low incidence and the great histological diversity of these tumors reduces the number of published articles about them. Indeed, most of the published reference series are retrospective studies, including cases diagnosed and treated for the last 20-30 years and giving mortality values that are far away from actual reality in pediatric hospitals [2, 3, 10-14]. Although prediction of the possible prognosis and postnatal outcome is complicated, perinatal, anesthetic and surgical
Pleuropulmonary blastoma. Axial fetal ultrasound (b, 22nd week) and fetal T2-WI (c, 24th week) demonstrate a well-defined, hypoechogenic, solid mass, isointense to lung on MRI (block arrow). Observe the pleural effusion (curved arrow)

care is constantly improving nowadays such that more infants survive in general.

In recent years, imaging methods have acquired a relevant role in the diagnosis of fetal tumors. Ultrasound is the standard method for fetal evaluation: it is ideal for screening, safe for mother and child, widely accessible and relatively inexpensive, but its effectiveness may be limited by factors such as oligoamnios, maternal body habitus, fetal position and fetal shadow bone artifacts. Prenatal MRI is increasingly being used as a complementary imaging technique, but its real utility for congenital tumors has not yet been widely evaluated. In general, fetal MRI should only be performed if it is considered that additional results might influence the management of the pregnancy and/or the therapeutic approach. Recent articles have shown a more accurate evaluation of MRI compared with ultrasound in some cases of congenital tumors, including the local anatomical extent of sacrococcygeal and cervicofacial teratomas, which may be underestimated on ultrasound because of fetal bone shadowing $[18,23]$. MRI may also discriminate between cystic and hemorrhagic areas with $\mathrm{T} 1$ or $\mathrm{T}^{*} *$ sequences, information that may be relevant for evaluating the therapeutic challenges for SCT $[18,23]$ and 
some intracranial tumors. MRI also seems to be better in the evaluation of the deep extension of large tumors that do not always respect anatomical planes, such as hemangiomas or cervicofacial teratomas [20,32]. Moreover, MRI allows a complete examination of the spinal canal in paravertebrally located tumors, such as neuroblastomas or SCT [23]. Finally, MRI performed during late pregnancy provides excellent anatomical details in a safe intrauterine environment, which may delay the need for immediate postnatal imaging.

Brain tumors are a special group of congenital neoplasms. The detection of a congenital brain tumor in a fetus is followed by serious ethical considerations about the real prognosis, postnatal quality of life, therapy challenges and long-term consequences of the applied therapy. The low incidence, great diversity and different behaviors of congenital brain tumors compared with tumors in older patients mean that published results, obtained from older children and adults, cannot be directly extrapolated. Overall prognosis is poor and therapeutic options limited, with an evident discrepancy between our diagnostic capacities and our therapy possibilities. A confident identification of the individual tumor types based on imaging is almost impossible, and therefore, in countries in which a legal medical interruption of pregnancy is possible, this diagnosis may lead to a high rate of legal abortions [44].

In conclusion, ultrasound and MRI have increased the rate of detection of congenital tumors in prenatal life. During pregnancy and after birth, the main role of imaging is to provide accurate information about the origin and the extent of the tumor, the invasion of adjacent organs, and the presence or absence of metastasis. This information is crucial for reducing the differential diagnosis and to evaluate and optimize the pre- and postnatal therapeutic challenges. A correct knowledge of the possibilities of the different imaging techniques in fetal and neonatal studies would improve all obtainable information, helping the medical team to make the most appropriate decisions about therapy. Prenatal ultrasound remains the standard diagnostic method, but fetal MRI seems to be an excellent complementary imaging technique, and its real utility should be evaluated in larger, multicentric series.

\section{References}

1. Woodward PJ, Sohaey R, Kennedy et al (2005) A comprehensive review of fetal tumors with pathologic correlation. Radiographics $25: 215-242$

2. Isaacs H (1985) Perinatal (congenital and neonatal) neoplasms: a report of 110 cases. Pediatr Pathol 3:165-210

3. Isaacs H Jr (1987) Congenital and neonatal malignant tumors: a 28-year experience at children Hospital of Los Angeles. Am J Pediatr Hematol/Oncol 9:121-129
4. Vassilatou-Kasmidis H (2003) Cancer in neonates and infants. Med Pediatr Oncol 41:7-9

5. Moore SW, Satgé D, Sasco AJ et al (2003) The epidemiology of neonatal tumors. Pediatr Surg Int 19:509-519

6. Parkes SE, Muir KR, Southern L et al (1994) Neonatal tumors: a 30-year population-based study. Med Pediatr Oncol 22:309-317

7. Barson AJ (1978) Congenital neoplasia: the society's experience. Arch Dis Child 53:436

8. Lornegan GJ, Schwab CM, Suarez ES et al (2002) Neuroblastoma, ganglioneuroblastoma and ganglioneuroma: radiologicpathologic correlation. Radiographics 22:911-934

9. Berbel O, Ortega JA, Ferris J et al (2008) Neonatal tumors and congenital malformations. An Pediatr (Barc.) 68(6):589-595

10. Isaacs H (2003) Fetal and neonatal leukemia. J Pediatr Hematol/ Oncol 25:348-361

11. Isaacs H (2004) Perinatal germ cell tumors. J Pediatr Surg 39:1003-1013

12. Isaacs H (2004) Fetal and neonatal cardiac tumors. Pediatr Cardiol 25:252-273

13. Isaacs H (2007) Fetal and neonatal hepatic tumors. J Pediatr Surg 42:1797-1803

14. Isaacs H (2008) Fetal and neonatal renal masses. J Pediatr Surg 43:1587-1595

15. Keslar PJ, Buck JL, Suarez ES (1994) Germ cells tumors of the sacrococcygeal region: radiologic-pathologic correlation. Radiographics 607-620

16. Brace V, Grant SR, Brackley KJ et al (2000) Prenatal diagnosis and outcome in sacro-coccygeal teratomas. Prenat Diagn 20:51-55

17. Altmann RP, Randolph JG, Lilly JR (1974) Sacrococcygeal teratoma: American Academy of Pediatrics Surgical Section Survey 1973. J Pediatr Surg 9:389-398

18. Avni FE, Guiband L, Robert Y et al (2002) MR Imaging of fetal sacrococcygeal teratoma: diagnosis and assessment. AJR Am J Roentgenol 178:179-183

19. Barksdale EM, Obokhare J (2009) Teratomas in infants and children. Curr Opin Pediatr 21:344-349

20. Vazquez E, Castellote A, Mayolas N et al (2009) Congenital tumors involving the head, neck and central nervous system. Pediatr Radiol 39:1158-1172

21. Hirose S, Farmer DL, Lee H et al (2004) The ex utero intrapartum treatment procedure: looking back at the EXIT. J Pediatr Surg 39:375-380

22. Wagner W, Harrison MR (2002) Fetal operations in the head and neck area: current state. Head Neck 24:482-490

23. Avni FE, Massez A, Cassart M (2009) Tumors of the fetal body: a review. Pediatr Radiol 39:1147-1157

24. Isaacs H (2007) Fetal and neonatal neuroblastoma: retrospective review of 271 cases. Fetal Pediatr Pathol 26:177-184

25. Biasotti S, Garaventa A, Villavecchia GP et al (2000) Falsenegative metaiodobenzylguanidine scintigraphy at diagnosis of neuroblastoma. Med Pediatr Oncol 35:153-155

26. Brodeur GM, Pritchard J, Berthold F et al (1994) Revisions of the international criteria for neuroblastoma diagnosis, staging and response to treatment. Prog Clin Biol Res 385:377-382

27. Brisse HJ (2009) Staging of common pediatric tumors. Pediatr Radiol 39(3):482-490

28. Cohn SL, Pearson ADJ, London WB et al (2008) The International neuroblastoma risk group (INRG) Classification system: an INRG task force report. J Clin Oncol 27:289-297

29. Mulliken JB, Fishman SJ, Burrows PE (2000) Vascular anomalies. Curr Probl Surg 37:517-584

30. Kamil D, Tepelman J, Berg C et al (2008) Spectrum and outcome of prenatally diagnosed fetal tumors. Ultrasound Obstet Gynecol 31:296-302

31. Boon LM, Enjolras O, Mulliken JB (1996) Congenital hemangioma: evidence of accelerated involution. J Pediatr 128:329-335 
32. Dubois J, Alison M (2010) Vascular anomalies: what a radiologist needs to know. Pediatr Radiol 40:895-905

33. Vilanova JC, Barceló J, Smirniotopoulos JG et al (2004) Hemangioma from head to toe: MRI with pathologic correlation. Radiographics 24:367-385

34. Castellote A, Vazquez E, Vera J et al (1999) Cervico-thoracic lesions in infants and children. Radiographics 19:583-600

35. Hayes-Jordan A, Andrassy R (2009) Rhabdomyosarcoma in children. Curr Opin Pediatr 21:373-378

36. Al-Nassan A, Sughayer M, Matalka J et al (2010) INI1 (BAF 47) immunohistochemistry is an essential diagnostic tool for children with hepatic tumors and low alfa-fetoprotein. J Pediatr Hematol/ Oncol 32:79-81

37. Bourdeaut F, Dufour C, Delattre O (2010) Rhabdoid tumors: hSNF/INI1 deficient cancers of early childhood with aggressive behaviour. Bull Cancer 97:37-45

38. Buetow PC, Smirniotopoulos JG, Done S (1990) Congenital brain tumors: a review of 45 cases. AJR Am J Roentgenol 155:587-593

39. Isaacs H (2002) Perinatal brain tumors: A review of 250 cases I. Pediatr Neurol 27:249-261

40. Isaacs H (2002) Perinatal brain tumors: A review of 250 cases II. Pediatr Neurol 27:333-342

41. Cassart M, Bosson N, Garel C et al (2008) Fetal intracranial tumors: a review of 2 cases. Eur Radiol 18:2060-2066

42. Sandow BA, Dory CE, Aguiar MA et al (2004) Congenital intracranial teratomas. Radiographics 24:1165-1170

43. Naeini RM, Yoo JH, Hunter JV (2009) Spectrum of choroid plexus lesions in children. AJR Am J Roentgenol 192:32-40

44. Rao P (2008) Role of MRI in pediatric neurooncology. Eur J Radiol 68:259-270

45. Panigrahy A, Blüml S (2009) Neuroimaging of pediatric brain tumors: from basis to advanced magnetic resonance imaging. $\mathrm{J}$ Child Neurol 24:1343-1365

46. Lowe LH, Ismani BH, Heller RM et al (2000) Pediatric renal masses: Wilms tumor and beyond. Radiographics 20:1585-1603
47. McNamara A, Levine D (2005) Intraabdominal fetal echogenic masses: a practical guide to diagnosis and management. Radiographics 25:633-645

48. Lonergan GJ, Martinez-Leon MJ, Agrons GA et al (1998) Nephrogenic rests, nephroblastomatosis and associated lesions of the kidney. Radiographics 18:947-968

49. Feng S-T, Chan T, Ching AS et al (2010) CT and MR imaging characteristics of infantile hemangioendothelioma. Eur J Radiol 76:e24-e29

50. Daller JA, Bueno J, Gutierrez et al (1999) Hepatic hemangioendothelioma: clinical experience and management strategy. J Pediatr Surg 34:98-106

51. Gembruch U, Baschat AA, Gloeckner-Hoffmann K et al (2002) Prenatal diagnosis and management of fetuses with liver hemangiomata. Ultrasound Obstet Gynecol 19:454-460

52. Hyett J (2008) Intra-abdominal masses: prenatal differential diagnosis and management. Prenat Diagn 28:645-655

53. Isaacs H (2010) Fetal and neonatal rhabdoid tumors. J Pediatr Surg 45:619-626

54. Wagner LM, Garrett JK, Ballard ET et al (2007) Malignant rhabdoid tumor mimicking hepatoblastoma: a case report and literature review. Pediatr Dev Pathol 10:409-415

55. Holley DG, Martin GR, Brenner JJ (1995) Diagnosis and management of fetal cardiac tumors: a multicenter experience and review of published reports. J Am Coll Cardiol 26:516-520

56. Wu SS, Collins MH, de Chadarevian JP (2002) Study of the regression process in cardiac rhabdomyoma. Pediatr Dev Pathol 5:29-36

57. Fesslova V, Villa L, Rizzuti T, Mastrangelo M et al (2004) Natural history and long-term outcome of cardiac rhabdomyomas detected prenatally. Prenat Diagn 24:241-248

58. Bussani R, Rustico MA, Silvestri F (2001) Fetal cardiac rhabdomyomatosis as a prenatal marker for the detection of latent tuberous sclerosis. An autopsy case report. Pathol Res Pract 197:559-561 